\title{
A Ku Band Circularly Polarized 2x2 Microstrip Antenna Array for Remote Sensing Applications
}

\author{
Roger Ferré, Fermín Mira, Guido Luzi, Jordi Mateu, Christos Kalialakis \\ Centre Tecnològic de Telecomunicacions de Catalunya (CTTC/CERCA) \\ Parc Mediterrani de la Tecnologia (PMT), Av. Carl Friedrich Gauss 7, 08860 - Castelldefels, Spain \\ christos.kalialakis@cttc.es
}

\begin{abstract}
This paper reports the development of a Circularly Polarized (CP) microstrip patch array working at $\mathrm{Ku}$ band and in particular between 16.9-17.2GHz for remote sensing applications. The design is based on a sequential rotation of four linearly polarized rectangular patches and appropriate phase shifts provided by a corporate feed network. The agreement between measurements and simulations carried out using HFSS is satisfactory.
\end{abstract}

Keywords-Microstrip; antenna; circular polarization; antenna arrays; remote sensing.

\section{INTRODUCTION}

The use of circular polarization(CP) in telecommunications, both in terrestrial and space borne applications, is well consolidated owing to its capability to maximize the polarization efficiency component of the link budget and its lower sensitivity to multipath clutter with respect to the linear polarized radiation.

The antenna developed in this work is intended to be used in a recent ground based microwave remote sensing application, namely Ground Based radar interferometry using Synthetic Aperture Radar(GBSAR)[1]. Printed antennas offer the possibility of low cost, low weight and scalability to high gain version[2] compared to conventional horn antennas.

Usually CP antenna arrays are composed of individual CP elements. Alternatively, linearly polarized (LP) elements can be used [3] which are in general easier to design than $\mathrm{CP}$ elements. The technique is based on imposing appropriate phase differences on each element in order to produce a composite CP field [4]. A $2 \times 2$ microstrip antenna array is developed here following this method.

\section{ANTENNA DESIGN}

\section{A. Design requirements}

The antenna array is intended as an antenna with a radar used for vibrations and deformations monitoring. The available in house radar device is a Continuous Stepped Frequency transceiver operating at $\mathrm{Ku}$ band. The apparatus is transportable and it is used in measurement campaigns in the field. Therefore lightweight components such as printed antennas are highly desirable. The frequency allocation for GBSAR[5] has a center frequency of $\mathrm{f}=17.1 \mathrm{GHz}$ and a total bandwidth of $300 \mathrm{MHz}(16.9-17.2 \mathrm{GHz})$. The antenna gain requirements are related to the distance of operation. Typical values for the antenna gain range from $10 \mathrm{~dB}$ to $20 \mathrm{~dB}$.

\section{B. Geometry and design procedure}

Exploiting the ideas in [3] and [4], four LP inset-fed half wavelength rectangular microstrip antennas are sequentially rotated(Fig.1a) and appropriate phase shift is applied on each branch of the feeding network (Fig.1b). The configuration for the case of Left Hand CP (LHCP) is shown in Fig.1. The design approach can be adjusted for Right Hand CP(RHCP) by employing rotations in the reverse sense.

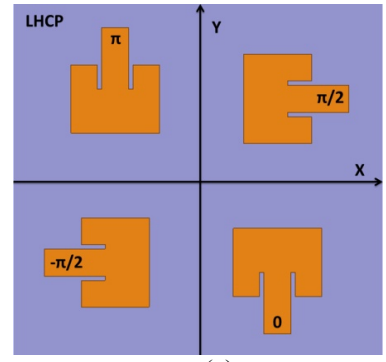

(a)

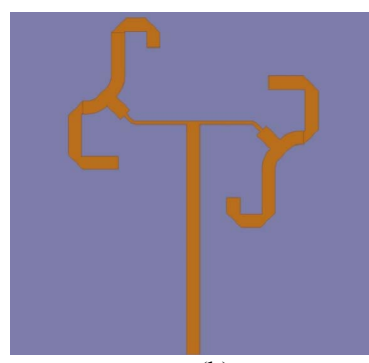

(b)
Fig. 1. (a) Sequentially rotated patches (b) Feeding network

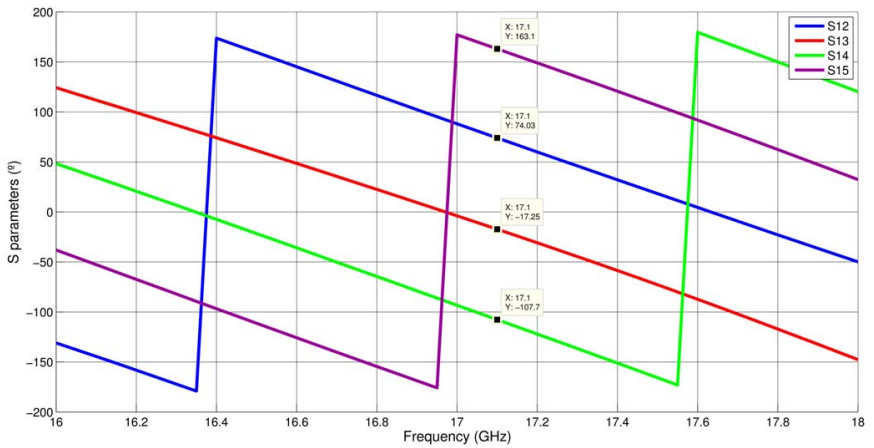

Fig. 2. Simulated S-parameters of the feeding network.

A critical point in the design procedure is the achievement of a proper $90^{\circ}$ phase difference among the four branches of the feeding network. As a first step, the feeding network was designed as a circuit in Keysight's Advanced Design System (ADS) and then optimized in Momentum to account for 
electromagnetic effects. In Fig. 2, the phase of the simulated Sparameters is plotted. The relative phase difference is $90^{\circ} \pm 1^{\circ}$ at the design frequency of $17.1 \mathrm{GHz}$.

Subsequently, the optimized feeding network along with the inset-fed antenna patches were simulated in HFSS. For the simulated antenna array, the calculated Axial Ratio(AR) remained below $2.5 \mathrm{~dB}$ in the frequency band of interest.

\section{ANTENNA MEASUREMENTS}

A prototype array was fabricated on an Arlon $25 \mathrm{~N}$ substrate $(\varepsilon \mathrm{r}=3.38, \mathrm{~h}=0.762 \mathrm{~mm})$ (Fig. 3) and measurements were carried out in an anechoic chamber.

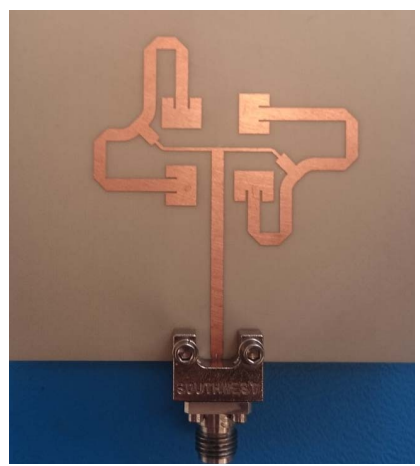

Fig. 3. The LHCP Antenna array prototype for the Ku band.

The measured impedance matching of the array is shown in Fig. 4. The return loss is better than $10 \mathrm{~dB}$ over a much wider bandwidth.

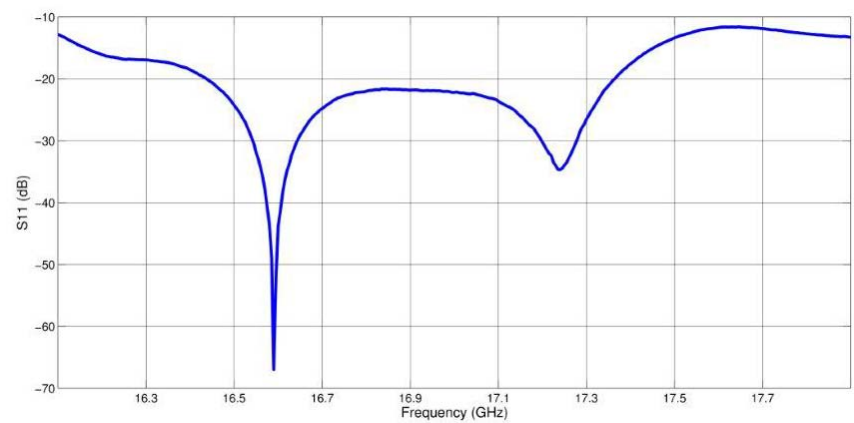

Fig. 4. Measured S11 of the LHCP antenna array.

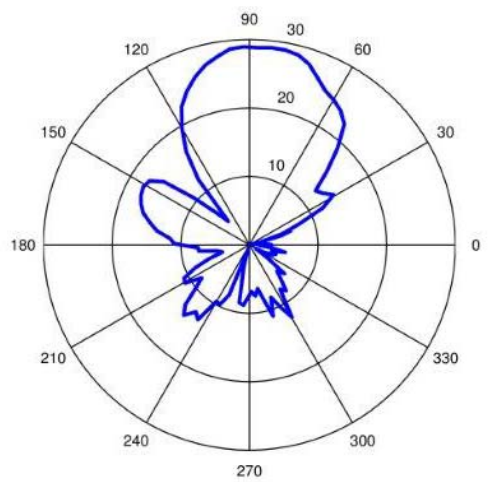

Fig. 5. Measured LHCP antenna pattern at $17.1 \mathrm{GHz}$
The measured antenna pattern at the center frequency is shown in Fig.5. If more gain is needed, the $2 \times 2$ antenna array can serve as a subarray for a larger array.

The most important metric for a CP antenna is its Axial Ratio. Measurements were carried out over frequency and angle (Fig. 6). As it can be seen, the measured broadside AR maintains values around $2.5 \mathrm{~dB}$ as predicted. The beamwidth is also stable.

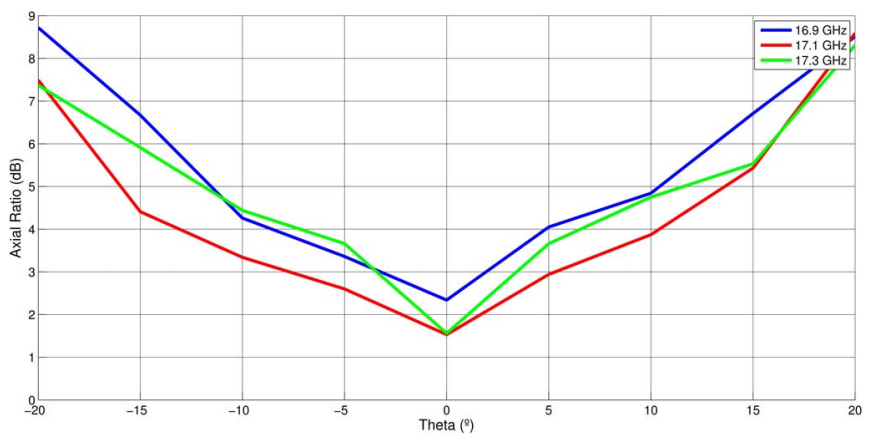

Fig. 6. Measured Axial Ratio over frequency and angle.

\section{CONCLUSIONS}

An LHCP 2x2 antenna array has been implemented for the $\mathrm{Ku}$ band by appropriate phase combinations of linearly polarized microstrip patches. The antenna array presents good impedance matching and axial ratio over the band 16.9-17.2 $\mathrm{GHz}$ showing promise as a good antenna candidate (in itself or as a subarray) for GBSAR applications in this band. Furthermore, the method can be adjusted to produce RHCP by reversing the sense of sequential rotation.

\section{ACKNOWLEDGMENTS}

The work has been supported by the Catalan Government under Grant 2014 SGR 1551. The work of C.Kalialakis was supported under EU H2020 Marie Sklodowska-Curie Grant Agreement 654734.

\section{REFERENCES}

[1] G. Luzi, "Ground-based SAR interferometry:a novel tool for geoscience", in Geoscience and Remote Sensing,New Achievements, P. Imperatore and D. Riccio(eds), Vukopvar:InTechopen, 2010, pp. 1-26.

[2] N. Boskovic, B. Jokanovic, F. Olivieri, D. Tarchi, "High gain printed antenna array for $\mathrm{FMCW}$ radar at $17 \mathrm{GHz}$ ", 12th International Conference on Telecommunication in Modern Satellite, Cable and Broadcasting Services, 2015.

[3] J. Huang, "A technique for an array to generate circular polarization with linearly polarized elements," IEEE Transactions on Antennas and Propagation, vol. 34, pp.1113-1124,1986.

[4] M. A., Rahman, Q. D. Hossain, M. A Hossain, E Nishiyama, I. Toyoda, "Design and parametric analysis of a planar array antenna for circular polarization", International Journal of Microwave and Wireless Technologies, vol.8, pp. 921-929, 2016.

[5] European Communications Committee, "Compatibility studies between Ground Based Synthetic Aperture RADAR and existing services in the range $17.1 \mathrm{GHz}$ to $17.3 \mathrm{GHz}$, Report 111,2007 . Online at http://www.erodocdb.dk/docs/doc98 /official/pdf/ECCRep111.pdf 